\title{
REVUE BIBLIOGRAPHIQUE SOMMAIRE DES TRAVAUX CONSACRÉS \\ A LA CORROSION MÉTALLIQUE EN LAITERIE
}

\author{
par \\ G. DAUFIN et M. SAINCLIVIER \\ Laboratoire de Recherches de Technologie laitière I.N.R.A. \\ Rennes
}

La corrosion est un des problèmes majeurs de l'industrie laitière tant sur le plan économique, que sur le plan technologique du maintien de la qualité des produits fabriqués.

Davis (1956) estime que négliger ce problème revient à réduire la durée d'utilisation du matériel de la moitié ou du quart du temps d'utilisation normale. La corrosion détériore les surfaces et les rend difficiles à nettoyer. Or le nettoyage et la désinfection du matériel de laiterie, que ce soit à la ferme ou à l'usine, représente une part importante des frais de récolte et de transformation du, lait. Pour assurer une production constamment de bonne qualité, il est nécessaire d'effectuer quotidiennement des circulations, trempages, pulvérisations d'eau froide ou chaude, de produits alcalins ou acides sur le matériel et l'équipement, dans les conditionnements, et les tuyauteries. Mais, la plupart des agents de nettoyage et de désinfection, de même que le lait ou les produits laitiers acides exercent sur les métaux une action corrosive appréciable. C'est un cercle vicieux dont il faut tenter de se dégager.

Mohr (1954), Davis (1956), Arup (1962) s'accordent à montrer l'importance de tous les problèmes concernant la corrosion, et l'intérêt qu'il convient de consacrer aux méthodes et techniques proposées pour l'éviter, le matériel de laiterie devenant de plus en plus coûteux.

Dans la présente publication nous proposons simplement une synthèse des connaissances acquises dans le domaine de la corrosion, en laiterie depuis la Deuxième Guerre mondiale sans prétendre à une étude exhaustive. Nous envisagerons successivement les travaux concernant les techniques d'étude et les divers facteurs d'influence sur la corrosion métallique.

\section{LES TECHNIQUES D'ETUDE DE LA CORROSION}

Toutes les techniques d'étude de la corrosion sont réunies dans le récent livre de Champion (1963). 


\section{Essais de corrosion dits "en service".}

Ils ont été utilisés avee succès par Flückiger et Heuscher (1965) sur des bidons à lait en service dans des fermes pour juger la tenue à la corrosion de l'anticorrodal (alliage à base d'aluminium connu en France sous le nom d'Almasilium) protégé par des couches d'oxyde d'épaisseur et de dureté différentes. Lindqvist et Magnusson (1966) ont effectué un travail identique. Camphausen (1961 a, b, et c) utilisé aussi ce type d'essais comme méthode de routine pour estimer la résistance à la corrosion de bidons soumis à l'action d'un certain nombre de cycles de nettoyage dans une machine à laver les bidons. C'est de cette manière que sont conçus les essais dits de longue durée pour l'agrément des produits de nettoyage par la DLG (1) en Allemagne fédérale (Kiermeier et al. 1965; Zühlsdorf, 1962 a).

Les renseignements recueillis sont exacts et précis mais les essais sont longs et ils ne permettent pas de mettre en évidence les causes de corrosion.

\section{Essais de laboratoire.}

Ils consistent à étudier les phénomènes de corrosion dans des conditions aussi proches que possible de celles rencontrées dans la pratique. Le principe de ces essais est décrit dans les ouvrages généraux de Akimov et Tomashov (1957), Champion (1963) et Uhlig (1963).

Botham et Dummett (1949) puis Botham (1962) ont utilisé des essais par immersion totale pour juger de la résistance à la corrosion des aciers inoxydables par les hypochlorites et les acides chloroisocyanuriques. Ils ont aussi effectué des essais par immersion partielle qui permettent de reproduire les conditions que l'on rencontre, en pratique, dans les tanks et les réservoirs à lait.

Actuellement, les essais par immersion intermittente ou immersion émersion alternées sont les plus employés. Mohr et Wortmann (1953) les ont utilisés pour évaluer la corrosion des métaux utilisés en laiterie par les solutions de nettoyage. Ils ont ensuite normalisé la méthode après avoir étudié l'influence de la qualité du métal, du traitement préalable de celui-ci avant l'essai, de la qualité de l'eau, de la durée d'action et du mode d'agitation. Le montage réalisé permettait de reproduire les cycles de nettoyage tels qu'ils se présentent dans la pratique industrielle, par exemple : trempages successifs dans une solution détergente et dans l'eau suivis d'un séchage. 700 cycles représentaient la valeur de deux années de travail dans une laiterie. Les opérations n'étant pas complètement automatiques, la méthode était fastidieuse. Elle a néanmoins permis à Zühlsdorf (1962 b) de déterminer l'intensité de la corrosion

(1) Deutsche Landwirtsehaft Gesellschaft. 
provoquée par 25 produits détergents et désinfectants du commerce sur le fer étamé et sur l'aluminium. Quinze seulement ont donné satisfaction. Pour chaque métal, une intensité maximum de corrosion (exprimée en perte de poids par unité de surface et par unité de temps) a été établie conventionnellement pour l'agrément des produits de nettoyage en Allemagne et pour l'attribution du label DLG. Plus tard Wildbrett (1958) et Wildbrett et Kiermeier (1960) ont construit un appareil entièrement automatique qui réalise des opérations identiques à celles utilisées par Mohr dans sa méthode manuelle. Les conditions des essais ont été normalisées ainsi que l'interprétation des résultats. Cette automatisation rend l'épreuve plus rapide et moins astreignante.

A l'aide d'un appareil conçu selon le même principe, nous avons montré (Daufin et Sainclivier, 1967) que les résultats des essais de corrosion sont reproductibles, mais qu'il est nécessaire de coupler les essais d'immersion-émersion avec des méthodes différentes (électrochimiques par exemple) pour prévoir la cinétique des phénomènes de corrosion.

Dés essais dits "aux vapeurs ", effectués par Botham et Dummett (1949) ont montré que dans certains cas (solutions iodées par exemple), la phase gazeuse est plus corrosive que la phase liquide.

Certaines techniques tiennent compte des facteurs mécaniques qui peuvent par érosion accélérer ou ralentir la corrosion. Etudiant plus particulièrement la corrosion des circuits de traite, Whittlestone (1963) a conçu un appareil d'essais accélérés qui permet de reproduire la corrosion subie par un métal sous l'action d'un détersif en circulation. Par exemple le détergent chaud circule dans une colonne verticale où sont placées les éprouvettes. Après un temps de repos pendant lequel les éprouvettes sont exposées à l'air, le détergent arrive de nouveau dans la colonne. La reproductibilité des résultats est satisfaisante.

Wildbrett et al (1961), ont examiné les différents facteurs intervenant dans la corrosion métallique consécutive au nettoyage et à la désinfection des tanks par pulvérisation sous pression. D'après Wildbrett (1963) les meilleurs résultats ont été obtenus par pulvérisation de vapeur sous de très fortes pressions. L'étude, au laboratoire, a été réalisée à l'aide d'un appareil automatique (Wildbrett et Kiermeier, 1966), dans lequel des éprouvettes métalliques subissent successivement l'action d'une solution détergente désinfectante, à des températures, des pressions et pendant des durées réglables selon les besoins de l'expérience.

Cet appareil, perfectionné et récemment bien mis au point, a permis à Kiermeier et al (1967) d'étudier le comportement des aciers inoxydables $18-8$ et l'aluminium 99,5 .

Tous ces essais ne donnent que des estimations globales quantitatives. En outre ils ne fournissent aueun renseignement sur la cinétique de la corrosion. Certains résultats peuvent se révéler insuffisants voire inexacts ainsi que le signale Defranoux (1966), 
en ce qui concerne le comportement des aciers inoxydables en milieu acide. Seules les méthodes électrochimiques peuvent, dans des cas semblables, donner des indications valables.

La corrosion est un phénomène électrochimique (Evans, 1929). Il était donc logique de penser à utiliser des méthodes électrochimiques qui ont l'avantage de donner des renseignements plus rapidement. Bien que l'interprétation des courbes soit relativement complexe, elles permettent généralement de définir un processus évolutif et les conditions dans lesquelles il prend naissance d'où la possibilité de prévoir la tenue à la corrosion d'un métal donné dans un milieu déterminé. Malheureusement, ces méthodes n'ont presque pas été utilisées dans les laboratoires de recherches laitières à l'exception de Rossmann (1964) qui a étudié la corrosion par piqûre de l'acier inoxydable austénitique 18-8 sous l'action de détergents-désinfectants combinés à base d'hypochlorite de Sodium.

Nous pouvons conclure ce chapitre concernant les techniques et leur utilisation pour l'étude de la corrosion en laiterie en faisant nôtre l'idée de Pourbaix et al (1963) : les auteurs estiment que l'utilisation combinée judicieuse des méthodes électrochimiques bien mises au point et des méthodes d'essais corrosimétriques bien adaptées au but recherché permet un contrôle industriel valable pour la plupart des cas de corrosion en présence de solutions aqueuses ou de milieux humides.

\section{LES FACTEURS INFLUENÇANT LA CORROSION}

Talbot (1967) rappelle que les phénomènes de corrosion mettent en présence deux partenaires : le métal et le milieu corrosif. L'attaque débute à la surface du métal, au contact du réactif et il est naturel d'envisager que l'état de surface, le réactif et le métal ont une influence sur la vitesse des phénomènes de corrosion.

\section{Influence de l'état de surface.}

L'influence de l'état de surface sur les phénomènes de corrosion rencontrés dans l'industrie laitière n'a pas été étudiée de façon systématique. Rappelons cependant que Noe (1964) a montré qu'il est plus intéressant de soigner l'état des surfaces en acier inoxydable $(17$ p. $100 \mathrm{Cr})$ que de chercher à utiliser des alliages nouveaux très onéreux (18-8 Mo) dont la tenue à la corrosion ne sera pas meilleure. Il souligne en outre l'influence primordiale de l'état de surface sur la résistance à la corrosion des aciers inoxydables.

\section{Influence des produits laitiers.}

Le lait frais et les produits laitiers non acides ont une action corrosive relativement faible sur les matériaux employés en laiterie (Mohr, 1954 - Arup, 1962). Cependant les produits acides (Gerhardt et Sommer, 1932 - Schwartze et al 1931), comme le lait écrémé acide et le sérum présentent déjà des propriétés corrosives impor- 
tantes. Toutefois, sur les aciers inoxydables (17 p. $100 \mathrm{Cr}, 18-10$ ) cette action est moins sévère que dans le cas d'une solution aqueuse d'acide lactique à même concentration, la présence de matières organiques a pour effet de diminuer l'agressivité de l'acide (Colombier et Hochmann, 1965 a). Davis, (1955) rappelle que les acides non oxydants tels que les acides lactique et eitrique peuvent être corrosifs à faible concentration. D'après Shreir (1963 a) l'acide lactique serait peut-être le seul élément important du lait responsable de la corrosion des métaux.

D'autres produits laitiers peuvent provoquer des cas de corrosion particuliers. Dans le cas du lait condensé, par exemple Summer (1965) a signalé une dissolution d'étain de l'ordre de $2 \mathrm{ppm}$, surtout si l'on conserve le lait dans la boîte ouverte. Jackson et al (1936 et 1937) ont montré en outre que les groupes - SH libres provenant de la crème après pasteurisation sont aussi la cause de certaines modifications de couleur du métal (brunissement). Stehle (1967) montre que le yoghourt aromatisé aux fruits provoque la corrosion des capsules d'aluminium. Il signale qu'un revêtement protecteur inerte de la face interne des capsules peut empêcher l'apparition de la corrosion.

En ce qui concerne le fromage, Flückiger (1964) a observé des boursouflures sous les emballages, en aluminium, de fromages fondus qui seraient dues à un dégagement d'hydrogène résultant de la corrosion de l'aluminium aux endroits où le vernis est défectueux. Szegö et Keresztessy (1964) ont montré que, lors du salage du fromage de brebis en sérum salé, les plaques d'acier étamé, quelle qu'en soit l'origine (Hongrie ou autres pays), se corrodent de la même manière et assez rapidement.

\section{Influence de la propreté des surfaces.}

Du point de vue de la corrosion, une surface propre est une surface exempte de toute particule solide étrangère. Or en laiterie on observe divers types de dépôts; pierre de lait, tartre, etc. Ces dépôts sont la cause d'une forme de corrosion qui relève du phénomène d'aération différentielle.

Walters et Vickers (1962) signalent que le lait peut être un facteur de corrosion, non seulement par certains de ses constituants chimiques mais aussi par la concentration de ceux-ci en un point donné; c'est le cas par exemple lorsqu'on laisse sécher un film de lait sur des parois métalliques. D'après Shreir (1963 a) le chauffage du lait forme sur les parois métalliques un film protecteur de colloïdes précipités.

Mais les produits laitiers et surtout le lait frais ou acide peuvent en outre déposer sur les parois avec lesquelles ils sont en contact, de la "pierre de lait" qu'il faut enlever par la suite. Si la pierre de lait est intéressante à connaître en raison de son influence sur l'apparition de la corrosion il n'est pas à propos de l'étudier ici. Le lecteur intéressé pourra se reporter aux récentes publications 
concernant sa formation (Whittlestone, 1961; Whittlestone et Murrane, 1962; Whittelstone et al, 1964; Roderig et al, 1956 ; Jensen, 1946 ; Levandowski, 1954 ; Lyster, 1965 ; Bell et Sanders, 1944 ; Johnson et Roland, 1940 ; Shere, 1942 ; Moore, 1944 ; Burton, 1961 et 1966) et concernant les procédés d'élimination (Shewing et Henderson, 1952; Kaufmann et al, 1955; Crossley, 1945 ; Parker, 1941 ; Levandowski, 1954; Morre, 1944; Holland et al, 1953 ; Ageev et al 1965 ; Scott et al, 1962 a et 1962 b ; Whittlestone, 1965).

L'ensemble de ces travaux montre l'importance que l'on attache à l'élimination des dépôts solides de lait séché ou chauffé. La première préoccupation a été d'éviter la formation de ces dépôts, mais lorsque aucune solution satisfaisante ne s'est présentée, il a fallu connaître la nature de la pierre de lait afin de choisir les produits d'élimination les plus efficaces possibles. Roderig et al (1956) par exemple ont effectué des essais en vue de trouver des agents suffisamment efficaces dans l'élimination de la pierre de lait sans pour autant être corrosifs envers les métaux utilisés en laiterie. Ils ont observé sur le matériel de ferme (manchons trayeurs, griffes, autolaveur, réfrigérant, canalisations) des types de pierre de lait différents requérant des détartrants différents et tous plus ou moins corrosifs. Bien qu'il ait paru difficile aux auteurs de donner un conseil en général, chaque cas particulier appelant sa propre solution, ils préconisent pour l'aluminium des solutions d'acide tartrique et pour l'acier inoxydable des solutions de sulfate de sodium.

\section{Influence de l'eau.}

Les principes de la corrosion par les eaux sont assez bien connus sur le plan théorique (Shreir, 1963; Poirier, 1963; Girard, 1964). Dans le domaine laitier, e'est seulement en tant que solvant des produits de nettoyage et de désinfection que l'eau a attiré l'attention des spécialistes. En effet, il est bien connu que la dureté de l'eau est d'une importance primordiale pour l'efficacité et le rendement des solutions de nettoyage. Il en est de même pour le rinçage et les dépôts dont elle peut être l'origine sont des causes de corrosion. Thiel (1958) dans une étude sur le nettoyage des trayeuses par immersion observe un abondant dépôt dû aux eaux dures très chaudes. Ces mêmes observations ont été confirmées par Major (1962) qui propose un détartrage périodique avec une solution chlorhydrique dans le cas des eaux trop dures. On peut songer à utiliser les eaux traitées de manière à les adoucir sans oublier que l'eau ayant subi un traitement non convenable est modifiée de telle façon qu'une corrosion importante peut en résulter ( $M c h r$, 1954). Aigner (1961) a essayé un produit à base de silicate de

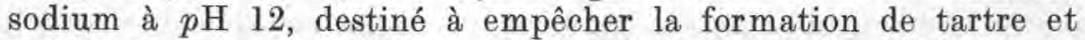
inhiber la corrosion par les eaux dans les tuyauteries et les échan- 
geurs thermiques. Il se forme sur les surfaces métalliques un film protecteur qui ne gène pas la transmission de la chaleur jusqu'à $100^{\circ}$ C. Au cours d'essais effectués durant douze mois consécutifs, Emerson (1962) trouve que le nitrite de sodium empêche la corrosion de l'acier doux dans un système de réfrigération à l'eau douce (alors qu'il n'y a naturellement pas de corrosion en eau dure) tandis que le traitement chlorure de calcium-bicarbonate de sodium est moins efficace.

\section{Influence des produits de nettoyage.}

Le passage quotidien de solutions de nettoyage sur le matériel laitier est une cause importante des cas de corrosion rencontrés dans l'industrie laitière. L'étude de la corrosion par les produits de nettoyage est difficile en raison de la multitude des produits employés, de leurs diverses compositions et des conditions d'utilisation très différentes d'un produit à l'autre, d'un type de matériel à un autre.

Les solutions fortement alcalines, de même que les acides forts sont des agents particulièrement corrosifs. Cependant (Mohr, 1954) il est possible d'employer des solutions acides si la durée d'action est courte. Toutefois, si l'acide nitrique pur a peu d'action (Davis, 1955) sur l'aluminium, il n'en est pas de même du même acide dilué à 1 p. 100 à chaud. Par ailleurs, si dans un nettoyage des machines à traire, par moussage d'un ammonium quaternaire, on intercale un nettoyage acide entre les deux traites, la corrosion augmente, que ce soit sur acier inoxydable, aluminium, cuivre étamé ou demi étamé (Whittlestone et Murrane, 1962). Il convient de préciser ici la nécessité d'employer un acide nitrique exempt de chlorures. Ce point est important parce que si l'acide nitrique par lui-même n'attaque pas l'acier inoxydable, la présence d'ion chlorure, même à froid, diminue la résistance à la corrosion (Colombier et Hochmann, 1965 b; Wotzilka, 1966). La concentration généralement recommandée est de 1 p. 100 (Swartling, 1959; Davis, 1965), la solution étant utilisée à $50^{\circ} \mathrm{C}$ pendant 20 à $30 \mathrm{minu}$ tes (Wolfrum, 1962). Mais les risques de corrosion dus aux impuretés de l'acide nitrique font que Botham (1949), Resuggan (1946) et Resuggan (1949) le déconseillent et donnent la préférence à des acides organiques tels que les acides gluconique, tartrique et citrique (Foster et al 1958, Whittlestone, 1958 et Whittlestone et Murrane, 1962).

Whittlestone (1962 b) a montré qu'un agent chélateur l'E.D.T.A. à raison de 5 p. 100 dans une solution détergente classique (hexamétaphosphate, métasilicate, soude, tensioactif) provoquait un accroissement marqué du taux de corrosion de l'étain et de l'aluminium.

On peut tirer parti dans l'industrie laitière de l'étude de Leclerc (1962) concernant l'influence des produits détergents sur la corro- 
sion des métaux par les eaux industrielles. On retiendra que les détergents anioniques après neutralisation ne présentent plus guère d'agressivité envers l'acier inoxydable, que les détergents non ioniques dilués sont peu corrosifs et que ce sont les impuretés de l'eau (surtout les chlorures) qui interviennent. Les détergents cationiques à réaction alcaline ne sont pas corrosifs.

II faut enfin signaler la corrosion consécutive à une mauvaise utilisation des produits de nettoyage (Peter, 1964; Mohr, 1954). Une température élevée entraîne presque toujours un renforcement de la corrosion, ainsi qu'un accroissement de la durée d'action, une concentration trop élevée, une pression trop forte (cas du nettoyage par jet).

\section{Influence des produits de désinfection.}

Le nettoyage ayant débarrassé les surfaces des salissures, la désinfection aura pour but de détruire les différents microorganismes qui s'y trouvent encore. Durant la totalité de leur action, les agents de désinfection agiront directement sur une surface métallique nue. L'accès à la surface des éléments corrosifs qu'ils contiennent sera donc particulièrement facilitée. Dans le cas d'utilisation de solutions détergentes-désinfectantes combinées, le produit agit comme agent de nettoyage d'abord, comme désinfectant ensuite. Les problèmes sont donc les mêmes bien que du point de vue de la corrosion la partie détergente peut modifier l'action du désinfectant, en agissant par exemple sur le $p \mathrm{H}$ de la solution.

Parmi les nombreux produits du commerce, on peut dire que les désinfectants contiennent comme éléments actifs, soit un halogène généralement le chlore ou l'iode, soit un dérivé halogéné (hypochlorite), soit un tensio-actif cationique, généralement un sel d'ammonium quaternaire. Ce sont les éléments halogènes qui sont le plus souvent responsables de l'apparition et du développement de corrosions parfois graves.

L'action corrosive du chlore et des dérivés chlorés.

Les hypochlorites attaquent les aciers inoxydables. Les produits de corrosion formés (oxyde noir de Nickel) catalysent la décomposition de la solution qui perd rapidement son chlore actif (Colombier et Hochmann, $1965 \mathrm{c})$.

Les solutions d'hypochlorite de sodium et de calcium provoquent facilement des piqûres profondes. Elles peuvent aussi occasionner une attaque sérieuse aux endroits où l'oxygène accède difficilement, par exemple dans les crevasses ou encore au niveau de surfaces métalliques en contact l'une avec l'autre (par exemple plaques de pasteurisateurs). L'acidification, même légère, des solutions désinfectantes aggrave sensiblement la corrosion. D'après Merrill et al (1962) la dissolution du chrome des aciers inoxydables 
par les solutions contenant du chlore est proportionnelle à la concentration en hypochlorite: Botham et Dummett (1949) avaient trouvé que les solutions commerciales de $\mathrm{Na} \mathrm{O} \mathrm{Cl}$ commençaient à être corrosives à la concentration de $150 \mathrm{ppm}$ de $\mathrm{Cl}_{2}$ actif à $40^{\circ} \mathrm{C}$ sur des métaux tels que l'aluminium, le cuivre étamé et même sur l'acier inoxydable 18-8 au molybdène. L'hypochlorite se décompose en $\mathrm{Na} \mathrm{Cl}$ et $\mathrm{Na} \mathrm{Cl} \mathrm{O}_{3}$ si bien que, dans les solutions, les rapports $\mathrm{Na} \mathrm{Cl} / \mathrm{Na} \mathrm{O} \mathrm{Cl}, \mathrm{NaCl} \mathrm{O} \mathrm{O}_{3} / \mathrm{NaO} \mathrm{Cl}$ et $\mathrm{Na} \mathrm{Cl} \mathrm{O}_{3} / \mathrm{Na} \mathrm{Cl}$ changent notablement dans le temps. Ce serait finalement les chlorures formés qui seraient responsables de la corrosion par piqûres. Rossmann (1964) montre que l'intensité de ce type de corrosion sur l'acier inoxydable $18-8$ est plus important à $40^{\circ} \mathrm{C}$ qu'à $20^{\circ} \mathrm{C}$ et à bas $p \mathrm{H}$ qu'à $p \mathrm{H}$ élevé. L'adjonction de $2 \mathrm{~g} / \mathrm{l}$ de silicate de sodium à une solution à 0,75 p. 100 d'eau de Javel suffirait à empêcher toute corrosion (Chave, 1956). Botham (1962) a essayé l'hypochlorite de sodium, les acides di- et tri- chloroisocyanuriques, le dichloro-isocyanurate de sodium et le I-3 dichloro 5-5 diméthylhydantoïne, à l'aide de quatre méthodes : immersion totale, immersion partielle, essai aux vapeurs, essai en condition de crevasses. Les deux dernières méthodes ont mis en évidence une corrosion notable plus importante par les acides di- et tri- chloroisocyanuriques que par l'hypochlorite. Les travaux de Bernhard (1962) montrent que les dérivés chlorés tels que la chloramine (à 200 ppm de chlore actif) sont plus corrosifs sur alliage d'aluminium et sur acier inoxydable que la formaline à 3 et 40 p. 100 en volume, que ce soit en solution agitée ou dans un courant continu de vapeurs saturées de désinfectant. Merrill et al, (1962) estiment que le phosphate chloré à $200 \mathrm{ppm}$ de chlore actif n'attaque pas l'acier inoxydable après immersion de 48 heures à $47^{\circ} \mathrm{C}$.

\section{L'action corrosive de l'iode et de ses dérivés.}

Les désinfectants iodés utilisés dans l'industrie sont presque exclusivement des composés appelés iodophores. Ils sont généralement associés à l'acide phosphorique. Johns (1954), dans ses premiers essais, n'observait qu'un taux négligeable de corrosion de l'acier inoxydable par les iodophores à $25 \mathrm{ppm}$ d' $_{2}$; plus tard (Johns, 1962) il n'observait aucune différence significative entre la corrosion provoquée par les iodophores et celle causée par un détergent alcalin chloré à $55 \mathrm{ppm}$ de $\mathrm{Cl}_{2}$. Les conclusions de Whittlestone et al (1964), sont identiques. Anderson (1962) confirme ces résultats tout en précisant que le cuivre et ses alliages sont attaqués. Davis (1962), par contre, a cité parmi les désavantages des iodophores leur effet plus corrosif que celui des détergents alealins à base d'ammonium quaternaire lors d'une utilisation sans précautions suffisantes (concentrations excessives, mauvais rinçages). En réalité Jensen et al (1963) montrent que, dans des conditionnements hermétiques traités aux iodophores, des dépôts d'iode se forment sur les parois: ces dépôts seraient le résultat 
de la condensation de la phase gazeuse s'évaporant des solutions d'iodophores. Ce sont eux les principaux responsables de la corrosion par piqûres ; c'est le cas par exemple dans les réservoirs réfrigérants de ferme qui restent fermés pendant que stagne la solution d'iodophore. Whittlestone et Lutz (1964) ont montré qu'une solution d'iodophore contenant 16 p. 100 de $\mathrm{H}_{3} \mathrm{PO}_{4}$ semblait légèrement plus agressive qu'une même solution ne contenant que 9 p. 100 de $\mathrm{H}_{3} \mathrm{PO}_{4}$. Whittlestone et Twomey (1966) ont confirmé leurs travaux déjà cités concernant le dépôt de pierre de lait et la corrosion; ils concluent que les meilleurs résultats sont obtenus en utilisant pour le prérinçage des installations de traite, des solutions d'iodophore ayant une teneur en acide phosphorique située entre 10 et 15 p. 100 . Enfin, parmi les avantages reconnus aux mélanges iodophores - acide phosphorique, Whittlestone (1965) cite tout particulièrement le pouvoir oxydant qui permet la conservation des couches protectrices d'oxyde sur les métaux.

\section{Influence des techniques de nettoyage.}

La manière dont la solution de nettoyage arrive au contact avec la surface des matériaux : solution non agitée, ou solution en circuit fermé à l'abri de l'oxygène de l'air ou encore emploi d'un jet de pulvérisation etc. est d'une importance considérable sur l'intensité de la corrosion (Mohr, 1954). Les techniques de nettoyage peuvent être classées en trois groupes:

- Immersion ou trempage nécessitant souvent un brossage mécanique ;

- Circulation pour laquelle Herreid et Le Roy Luetscher (1963) ont montré que le taux de corrosion est proportionnel à l'acidité des produits laitiers, à la dureté, à l'acidité ou à l'alcalinité des eaux, à la température, à la teneur en oxygène, éte. ;

Les phénomènes de turbulence constituent un facteur de corrosion supplémentaire (Whittlestone, 1958; Scott et al, 1962).

- Pulvérisation utilisée pour le nettoyage des grands conditionnements.

Ces dernières années les recherches porte principalement sur les récentes techniques automatiques. Wildbrett (1962) pensait que les nouvelles techniques de pulvérisation pour le nettoyage des tanks accroissaient les dangers de corrosion et qu'il convenait d'envisager l'emploi d'aciers inoxydables de nouvelle composition. Aussi à l'aide d'appareils conçus spécialement pour ces recherches (Wildbrett et Kiermeier, 1966), Wildbrett et al (1962), Kiermeier et al (1963), Wildbrett (1964), Kiermeier et al (1967), étudient les techniques par injection de vapeur (efficace mais lent refroidissement ultérieur), par pulvérisation à basse pression, ainsi qu'un procédé de nettoyage acide suivi d'un nettoyage alcalin sans rinçage intermédiaire (pour les pasteurisateurs par exemple) mais il ne semble pas que les 
résultats obtenus quant à leurs avantages ou inconvénients du point de vue de la corrosion permettent encore une interprétation transposable dans le domaine de la pratique.

\section{L'INFLUENCE DE LA NATURE DES MÉTAUX UTILISÉS EN LAITERIE}

Dans une étude de synthèse sur les emballages alimentaires Lefaux (1960) rappelle que quatre métaux "alimentaires» ou alliages sont essentiellement utilisés en laiterie : l'aluminium et ses alliages tels que l'almasilium ( $\mathrm{Al}, \mathrm{Mg}, \mathrm{Si}$ ), le fer étamé et l'acier inoxydable. Sanderson (1964 et 1966) passe périodiquement en revue les progrès réalisés en métallurgie examinés du point de vue de leurs applications dans l'industrie laitière. Ses récentes publications portent essentiellement sur les aciers inoxydables de nouvelle composition, sur les nouveaux alliages d'aluminium (Al-Mg$\mathrm{Zn}, \mathrm{Cu}-\mathrm{Al}-\mathrm{Zn}$ ), sur l'allègement du poids des réservoirs, sur les caractéristiques de résistance à la corrosion, etc. Actuellement l'acier inoxydable est le plus employé des métaux en laiterie. Le type En $58 \mathrm{~J}$ (norme anglaise $=\mathrm{Cr} 18-\mathrm{Ni} \mathrm{10}$, Mo 2,75, Mn 0,8, Si $0,3, \mathrm{C} 0,07$ ), c'est-à-dire ce que l'on appelle plus couramment l'acier inoxydable au molybdène, n'est nécessaire que dans le cas de produits laitiers très acides (Botham, 1953). L'aluminium est également très utilisé mais il est sensible à la corrosion par piqûres, surtout aux températures élevées (Aziz et Goddard, 1952). L'étain et le cuivre sont utilisés là où ils ne peuvent être préjudiciables à la qualité des produits laitiers. Davis, (1956) donne un classement des métaux dans l'ordre de leur résistance décroissante à la corrosion en laiterie : acier inoxydable, étain, cuivre étamé, nickel, aluminium et ses alliages, fer étamé, cuivre et fer galvanisés, fer, zinc. Ces quatre derniers métaux sont d'ailleurs fortement déconseillés et ne sont plus utilisés pratiquement.

\section{Corrosion de l'acier inoxydable.}

Trois types d'acier inoxydable sont utilisés en laiterie : l'alliage austénitique 18-8 et les alliages martensitique et ferritique qui ne contiennent pas de Nickel (Herreid et Le Roy Luetscher, 1963). Davis (1956), Rogner (1958) puis Ameen et Anderson (1962) décrivent les divers types de corrosion et leurs conditions d'apparition sur l'acier inoxydable.

Les solutions acides, notamment chlorhydrique et formique et même nitrique ou phosphorique en eau légèrement chlorurée, sont des agents actifs de corrosion uniforme. Dans tout le matériel de laiterie, il faut, selon Chave (1956) préférer un acier 18-8 à bas carbone en raison de sa résistance aux acides du lait et à l'acide nitrique de nettoyage.

Wolfrum (1962) rappelle à juste titre que la résistance des aciers inoxydables à la corrosion dépend avant tout de la passi- 
vité de la surface mais Cunha Belo et al. (1964) précisent que l'étude seule des propriétés des films passivants ne permet pas de prévoir le comportement des aciers aux divers genres de corrosion. Ils ont montré que le degré de pureté, la structure du métal (dépendant elle-même non seulement de la composition mais du traitement thermique) sont en relation avec la plus ou moins grande sensibilité à la corrosion.

Les chlorures (présents dans la pierre de lait, dans les impuretés des solutions acides de nettoyage, dans les eaux, dans les produits de décomposition du $\mathrm{Cl} \mathrm{O} \mathrm{Na}$, etc.), sont agressifs envers l'acier inoxydable surtout aux $p H$ neutres ou acides. Le $p H$ doit être égal ou supérieur à 8 . Colombier et Hochmann $(1965 \mathrm{~b})$ rappellent qu'il est prudent d'éviter le séchage des solutions chlorurées à la surface des métaux pendant la période de non utilisation des appareils, les cristaux augmentant les risques de corrosion. Certaines eaux sont riches en chlorures et il faudra éviter de les utiliser notamment pour diluer les acides servant à ce type de nettoyage.

Botham et Dummett (1949) avaient mis en évidence l'influence de la température et du $p \mathrm{H}$ ainsi que celle de la durée de contact. Ils avaient trouvé en outre que l'acier 18-8 à 3 p. 100 de Molybdène était plus sensible à la corrosion par piqûres que le $18-8$ à 6 p. 100 de Titane. Botham (1962) confirme cette dernière observation dans le cas de piqûre provoquée par les hypochlorites.

Des cas de corrosion sous tension ont été observés dans les réfrigérateurs à lait; ceux-ci constituent des empilements en tôles d'acier 18.8 entre lesquelles circulent en couches alternées le lait et la saumure. Les fissurations se localisent sur la face convexe des parties embouties et d'après Nathorst (1950) seraient dues à l'action de traces résiduelles de chlorures. Wotzilka (1966) a observé les mêmes phénomènes. La corrosion sous tension apparaît très rarement dans les systèmes contenant de l'eau d'extrême pureté mais quelques $\mathrm{ppm}$ de $\mathrm{Cl}^{-}$peuvent suffire pour provoquer cette corrosion (Williams et Eckel, 1956).

Les cas de corrosion galvanique sont devenus relativement moins fréquents aujourd'hui où l'équipement laitier est presque toujours en acier inoxydable. Toutefois, lorsque, par exemple un acier au $\mathrm{Ni}$ et un acier sans $\mathrm{Ni}$, subissent simultanément le contact d'une même solution de nettoyage (cas du "cleaning in place " par exemple) l'acier sans nickel peut se corroder (Walters et Vickers 1962). Ce phénomène est confirmé par Herreid et Le Roy Luetscher, 1963.

La corrosion intergranulaire se rencontre peu fréquemment à l'heure actuelle. Les aciers au Titane ou au Nobium et les aciers à bas carbone $(<0,02$ p. 100) ne sont pas sensibles à ce type de corrosion (Davis, 1955 et 1965), (Colombier et Hochmann, 1965).

Les aciers inoxydables représentent finalement le matériel le plus approprié comme matériel de laiterie que ce soit à la ferme 
pour les canalisations de machine à traire (Whittlestone, 1962 a) ou pour l'équipement des usines laitières.

\section{Corrosion de l'étain.}

L'étain est surtout utilisé comme métal de protection sous réserve que la couche utilisée soit non poreuse et d'environ $50 \mu$ d'épaisseur (Davis, 1956). La solubilisation de l'étain par les alcalis est réduite par adjonction de sulfite de soude dans les lessives. Les acides oxydants tel que l'acide nitrique sont très destructeurs. Même l'acide phosphorique, peu agressif en milieu non aéré, le devient en milieu aéré. Au cours d'essais de détartrage sur le fer étamé absolument lisse Lewandowski (1952) trouve que le fer étamé obtenu par galvanoplastie est plus facilement attaqué que celui obtenu par trempage en bain chaud d'étain. Adams et al. (1965), après quatre années d'essais en service de boites étamées contenant du lait concentré non sucré, n'observent pas de différence significative entre les teneurs en $\mathrm{Sn}$ et en $\mathrm{Fe}$ du lait par rapport à celles du lait contenu dans des boîtes identiques récemment remplies. Par contre le goût du lait serait modifié. On utilise de moins en moins le fer étamé pour les bidons et la vaiselle laitière en raison des difficultés de nettoyage, de sa toxicité à partir d'une certaine concentration et de son peu de résistance mécanique (Davis, 1956). Toutefois des bidons en acier étamé sont encore très utilisés notamment en Angleterre où ils doivent être conformes aux normes de la British Standard (1948) concernant par exemple les dimensions, les doses limites de solubilité dans le lait et les produits détergents.

\section{La corrosion du cuivre et du cuivre étamé.}

Les produits de corrosion qui n'auraient pas été éliminés par un rinçage suffisant passent dans les produits laitiers en provoquant diverses altérations. Leur saveur, leur odeur et leur couleur peuvent être affectées par des traces d'ions métalliques (Lefaux, 1960). Le fer et le cuivre communiquent fréquemment des saveurs métalliques.

De nombreux chercheurs ont étudié le rôle catalyseur des traces de cuivre sur les goûts d'oxydé et les saveurs métalliques qu'elles provoquent dans les produits laitiers mais cela dépasse le cadre de cette revue bibliographique. Ces goûts métalliques apparaissent dans le lait dès que les teneurs en cuivre, fer ou zinc dissous dépassent $1 \mathrm{ppm}$ selon Davies (1932). Le cuivre peut être amené dans le beurre par les eaux; Swartling (1956) a observé que du cuivre amené par l'eau chaude ayant circulé dans les tuyauteries des chaudières se dépose sur les parois de l'équipement laitier et contamine le beurre. Durkley et King (1959) ont montré que, lorsqu'on fait passer une solution de nettoyage sur une pièce en 
alliage de cuivre, une partie de ce métal passe en solution et se dépose ensuite dans le circuit.

En fromagerie de Gruyère, au contraire, des traces de euivre seraient bénéfiques en inhibant le développement des ferments butyriques, agents de gonflement. C'est pourquoi les cuves en acier inoxydable sont abandonnées dans certains cas pour revenir au cuivre, ou recouvertes de cuivre par dépôt électrolytique.

Dans l'ensemble, le cuivre et ses alliages sont de plus en plus délaissés sauf en fromagerie de Gruyère. Scott et al. (1962 a), Whittlestone et Lutz (1962 a et b et 1963) ont effectué différentes recherches qui visaient à réduire la contamination du cuivre due à la corrosion par les détergents ; l'étamage par dépôt électrolytique d'un alliage Sn-Ni s'est révélé plus résistant à la corrosion que l'étamage à l'étain seul et plus facile à nettoyer. Ils ont constaté que lors de la dissolution de la pierre de lait par différents détergents préparés à cet effet, le cuivre à demi étamé est plus fortement attaqué que le cuivre étamé. Le cuivre, étant encore utilisé en fromagerie, il serait peut-être intéressant de voir si l'on ne pourrait pas tirer profit en laiterie des travaux de Serre et Laureys (1963) sur l'amélioration constatée par l'utilisation du euivre passivé par oxydation à chaud. Pourbaix et al. (1963) observent de leur côté qu'une faible addition de $\mathrm{Na} \mathrm{H} \mathrm{CO}_{3} \quad(0,03 \mathrm{M}$ soit 2,5 g par litre) à l'eau distillée provoque une passivation du cuivre alors qu'une forte addition $(0,1 \mathrm{M}$ soit $8,4 \mathrm{~g}$ par litre) provoque une corrosion. Whittlestone et Lutz (1962 a) enfin, ont trouvé que l'addition d'un dérivé de l'E.D.T.A. aux solutions de nettoyage augmente la corrosion du cuivre et de l'aluminium.

\section{La corrosion de l'aluminium et de ses alliages.}

Tous les mélanges détergents utilisés sur l'aluminium devront contenir au moins 25 p. 100 de silicate de sodium pour éviter la corrosion. Toutefois d'après Whittlestone et Lutz (1962 a) cinq détergents du commerce à $p \mathbf{H}$ basique corrodent l'aluminium bien qu'ils contiennent 25 p. 100 de silicate de sodium. Lindqvist et Magnusson (1966) montrent que $\mathrm{Si} \mathrm{O}_{2}$ joue le rôle de colloïde protecteur empêchant le dépôt de silicate de calcium et le vrai facteur de corrosion ou d'inhibition serait alors le rapport $\mathrm{Na} \mathrm{OH} /$ $\mathrm{Si}_{2}$. Krüger et al. (1962) ont constaté qu'il n'y a jamais de corrosion de l'aluminium tant que le rapport $\mathrm{Na} O \mathrm{OH} / \mathrm{Si} \mathrm{O}_{2} \leqslant 2,5$ à $40^{\circ} \mathrm{C}$ et $65^{\circ} \mathrm{C}$ et qu'il y a corrosion intense lorsque ce rapport est égal ou supérieur à 17,5 à $40^{\circ} \mathrm{C}$ ou égal ou supérieur à 6,5 à $65^{\circ} \mathrm{C}$. D'après Lindqvist et Magnusson (1966) un détergent à $p \mathrm{H}$ élevé contenant 40 p. 100 de métasilicate, 20 p. 100 d'orthosilicate et 39 p. 100 de triphosphate (tous sels de sodium) n'attaque pas l'aluminium, à haute température (supérieure à $80^{\circ} \mathrm{C}$ ). Il semble que la température favorise une passivation par formation d'oxyde. Par contre Lindqvist et Magnusson (1963) trouvent une corrosion 
intense à $40^{\circ} \mathrm{C}$ et $60^{\circ} \mathrm{C}$. L'aluminium pur est fortement attaqué par $\mathrm{Na}_{2} \mathrm{CO}_{3}$ ou par $\mathrm{Na}_{3} \mathrm{PO}_{4}$. L'examen des courbes d'attaque de l'aluminium par des mélanges de silicate et de phosphate trisodique ou de carbonate de soude (Degeorges, 1960) montre que pour observer un minimum d'attaque, le pourcentage en métasilicate à introduire augmente avec la dilution d'emploi, une certaine concentration en silicate devant toujours être maintenue.

Bien que la corrosion uniforme soit couramment observée (Zühlsdorf, 1962 b) elle est le plus souvent accompagnée de piqûres, notamment en présence de solution d'hypochlorite à $150 \mathrm{ppm}$ de $\mathrm{Cl}_{2}$ actif (Botham et Dummett, 1949). Bailley et Brace (1956) n'ont pas constaté de corrosion notoire sur des bidons en alliage d'aluminium en service, nettoyés à l'eau chaude puis désinfectés à l'aide d'une solution chaude d'hypochlorite à $300 \mathrm{ppm}$ de $\mathrm{Cl}_{2}$ actif.

La protection de l'aluminium est obtenue, industriellement par oxydation anodique. L'alumine formée est douée de remarquables propriétés anti-corrosives et d'isolement thermique et électrique, et est en outre plus dure que l'aluminium lui-même et lui assure une protection mécanique (Wrzeclan, 1964 et Darnault, 1965). Une telle protection par oxydation anodique serait satisfaisante à l'usage pour les bidons de lait (Flückiger, 1961). La couche d'alumine peut aussi être obtenue par oxydation chimique (Hess, 1951 ; Meynis de Paulin, 1957). A titre indicatif, la couche d'alumine naturelle par oxydation à l'air serait de l'ordre de $1 / 100$ à $3 / 100$ de micron, de 1 à 3 microns dans le cas d'oxydation par voie chimique et de 2 à 25 microns par voie électrolytique (Lefaux, 1960). Lorsque le film est enlevé par une cause quelconque, le métal est vulnérable et n'est à nouveau protégé que par une nouvelle formation d'alumine. Cette nouvelle formation naturelle n'est pas encore complète en 24 heures (Davis, 1955). Flückiger (1961), Flückiger, (1962) et Flückiger et Heuscher (1965) ont étudié un mode de protection des bidons en anticorrodal ( $\mathrm{Al}, \mathrm{Mg}, \mathrm{Si}$ ) contre la corrosion due à des traces de cuivre que l'on rencontre en fromagerie. Trois types de couches d'oxyde (épaisse, dure, dure et épaisse) obtenues par des conditions différentes d'oxydation anodique ont été éprouvées. La différence de la tenue à la corrosion de ces trois couches n'a pas été appréciable après une année de service. Szegö et Keresztessy (1959) ont soumis des plaques d'aluminium à l'épreuve de l'immersion totale dans différents liquides: lait frais ou acide, solutions détergentes alcalines ou acides, saumures etc. Ils ont comparé l'Al poli non traité et des plaques traitées anodiquement de manière à avoir des épaisseurs de couche d'oxyde de $6 \mu, 10 \mu$ et $13 \mu$. Leurs résultats semblent différer de ceux d'autres chercheurs puisqu'ils constatent une perte de poids d'autant plus marquée que la couche d'alumine est plus épaisse. Ces différences sont peut-être imputables au fait que les différents auteurs n'ont pas utilisé les mêmes méthodes. De plus nous ne 
sommes pas renseignés sur la porosité des couches d'alumine, porosité qui joue certainement un rôle important dans la résistance à la corrosion.

Flückiger et Heuscher (1965) estiment que les alliages d'aluminium de type anticorrodal méritent d'être conservés car ils présentent de gros avantages: légèreté, solidité, aspect agréable. C'est aussi l'opinion de Kohn-Abrest (1949) à condition que le nettoyage quotidien soit effectué soigneusement et avec des produits convenant bien au métal.

\section{La corrosion du fer étamé.}

Zühlsdorf (1962 b) a mis en évidence la corrosion par piqûre que subissent les bidons en fer étamé. Ils sont pratiquement abandonnés maintenant et fortement déconseillés par Davis (1956) et Mohr (1954).

\section{La corrosion du zinc.}

Davis (1955 et 1965) rappelle que le zinc, s'il est utilisé en laiterie pour revêtement de matériel en acier doux ou en fer n'est pas de toute manière utilisé lorsqu'il doit y avoir contact avec le lait. En raison de la toxicité des traces de zine, Truffert (1949) a proposé l'interdiction de l'emploi de matériel galvanisé en laiterie.

\section{CONCLUSION}

Il ressort de cette étude bibliographique qu'un certain nombre de résultats importants ont déjà été acquis :

- Les matériaux ne présentant qu'une résistance convenable à la corrosion ont été éliminés. C'est le cas du fer étamé, du zine, du cuivre (à quelques exceptions près en fromagerie) et de l'étain.

- Pour conserver le bénéfice d'autres avantages, ou pour éviter de trop importants investissements en matériaux nouveaux, des moyens de protection ont été étudiés et sont déjà utilisés en pratique (oxydation anodique de l'aluminium, étamage du cuivre, revêtement en alliage étain nickel...).

Cependant l'utilisation de plus en plus répandue des aciers inoxydables, de matériaux nouveaux (Titane) rend nécessaire dès maintenant l'étude approfondie des phénomènes de corrosion et il semble que les chercheurs spécialisés dans la corrosion en laiterie doivent contribuer à résoudre certains problèmes fondamentaux afin de trouver les moyens de lutter, par exemple, contre la corrosion par piqûre des aciers inoxydables (le chlore et les chlorures étant à des titres divers des agents très utilisés en laiterie : désinfection, salage, préparation des saumures pour maintien des basses températures etc.).

Du point de vue expérimental, il semble urgent de normaliser les essais de corrosion et de les adapter à la pratique. Il ressort en effet des travaux déjà effectués qu'il est fort difficile d'émettre une conclusion générale dans un cas donné, car les auteurs ont effectué leurs essais dans des conditions différentes (ce qui explique 
peut-être les divergences de résultats dans certains cas), ils ne définissent pas toujours clairement la composition et "l'histoire " des matériaux. Enfin les résultats d'essais de laboratoire ne peuvent pas toujours être transposés sans réserve dans la pratique et des prévisions hâtives peuvent conduire à de grandes déceptions.

Il serait intéressant de développer l'emploi des méthodes d'étude électrochimiques et de les adapter aux conditions de corrosion rencontrées en laiterie afin de mieux cerner les problèmes et leur résolution. A cet effet un contact étroit entre spécialistes de la science de la corrosion dans les domaines fondamentaux et les spécialistes de la technologie laitière sera le meilleur moyen d'entreprendre un programme de recherche constructif et efficace.

\section{Summary}

This bibliographic study of one hundred french and foreign works shows that:

- The materials which do not afford a right corrosion resistance have been strike off, such as timed iron, zine, copper (with some exceptions in cheese-making) and tin.

- To conserve the profit of other advantages, or to forbid too many investings of new materials, means of protection hase been studied and are already used in practice (anodic oxidization of aluminium), tinning of copper, coating alloying tin-nickel.

Yet more and more spread utilization of stainless steels, of new materials (titanium) makes necessary already a thorough study of dairy corrosion phenomenons.

It seems that specialized seekers in dairy corrosion must cooperate in resolving eertain fundamental problems, in order to find means of struggling against, for example, pitting corrosion of stainless steels (chlorine and chlorides beeing in different claims determining elements very used in dairy: disinfection, salting, picking brine préparation for keeping low temperatures...).

On an experimental point of view it seems instant to normalize corrosion tests and to adapt them to practice.

Nous remercions très vivement $M$. G. Mocquot, directeur de la Station centrale de Recherches laitières (I.N.R.A. Jouy-en-Josas) et M. Talbot, professeur à l'Ecole nationale supérieure de Chimie (Paris) pour avoir bien voulu nous aider de leurs précieux conseils dans ce travail.

\section{BIBLIOGRAPHIE}

Adams (H. W.), Hartman (G. H.), Oberg (E. B.), Pearoe (S. J.), Greiner (F. J.) et Parfitt (E. H.). 1965. J. Dairy Sci., 48, 425-430.

Ageev (L.) et Novotel'Nova (N.). 1965, en russe, Mol. Prom., 26, 32-34. AIGNER (M.). 1961, Osterr. Milchw., 16, 66-68.

Akimov (G. V.) et Tomashov (N. D.). 1957, Théorie et méthodes d'essai de la corrosion des métaux, Dunod, Paris.

Ameen (L.) et Andersson (B.). 1962, Die Stärke, 14, 118-124.

ANDERSON (L.), 1962, Milchw., 17, 513-517.

ARUP (H.). 1962, Milk Ind., 51, 41-43. 
Aziz (P. H.) et Goddard (H. P.). 1952, Ind. Engineer. Chem. (Indus.), 44, 1791.

Bailley (J. C.) et Brace (A. W.). 1956, J. Soc. Dairy Technol., 9, 155-159.

Bell (R. W.) et SANDers (C. F.). 1944, J. Dairy Sci., 27, 499-502.

Bernhard (E.). 1962, XVIe Cong. Int. Lait., $C, 141-145$.

Вотнам (G. H.), 1949, XII e Cong. Int. Lait., 3, 310-314.

Вотнам (G. H.). 1953, J. Soc. Dairy Technol., 6, 179-187.

Вотнам (G. H.). 1962, XVIe Cong. Int. Lait., $C, 167-175$.

Botham (G. H.). et Dummett (G.A.). 1949, J. Dairy Res., 16, 23-38.

British Standard Specification (1948), Performance tests for protective schemes B.S. 1484.

Burton (H.). 1961, J. Dairy Res., 28, 255-263.

Burton (H.). 1966, J. Dairy Res., 33, 317-324.

Camphausen (H.). 1961 a, Molk. und Käs. Ztg., 12, 774-777.

Camphausen (H.). 1961 b, Molk. und Käs. Ztg., 12, 836-837.

Camphausen (H.). 1961 e, Molk. und Käs. Ztg., 12, 907-910.

Champion (F.A.). 1963, Corrosion testing procedures, $2^{\mathrm{e}}$ ed., Chapman et Hall, London.

Chave (P.). 1956, Ind. Alim. Agric., 73, 869-870.

Colombier (L.) et HochmanN (J.). 1965 a, Aciers inoxydables, aciers réfractaires, 315, 2e éd., Dunod, Paris.

Colombier (L.) et Hoohmann (J.). 1965 b, Aciers inoxydables, aciers réfractaires, 317-318, $2^{e}$ éd., Dunod, Paris.

Colombier (L.) et Hochmann (J.). 1965 c, Aciers inoxydables, aciers réfractaires, 320, $2^{\mathrm{e}}$ éd., Dunod, Paris.

Crossley (E. L.). 1945, J. Dairy Res., 14, 160-164.

Cunha Belo (M.), Berge (P.), Montuelee (J.) et Chaudron (G.). 1964, Corr, et Antic., 12, 297-307.

Darnault (G.). 1965, Prat. Indus. mécan., 48, 305-308.

Daufin (G.) et Sainchivier (M.). (sous presse, Corr.).

Davies (W. L.). 1932, J. Dairy Res, 3, 86-92.

Davis (J. G.). 1955, A dictionary of Dairying, $2^{\mathrm{e}}$ éd., Leonard Hill Ltd. London.

Davis (J. G.). 1956, Laboratory control of Dairy plant, Dairy Ind, Ltd., London.

Davis (J. G.). 1962, J. Appl. Bact., 25, 195-201.

Davis (J. G.). 1965, The supplement to A dietionary of Dairing, Leonard Hill Ltd, London.

Defranoux (J. M.). 1966, Corr. et Anticor., 14, 270-273.

Degeorges (M.). 1960, Etude des détergents, Ed. Cebedoc, Liège.

DURKLEY (W. L.) et King (R. L.). 1959, J. Dairy Sci, 42, 480-488.

Emerson (A. G. D.). 1962, Dairy Engineer., 79, 431-435.

EVANS (U. R.). 1929, J. Franklin Inst., 208, 45-48.

FlÜCKIGER (E.). 1961, Schweiz. Milchztg., 87, 117.

FLÜCKIGER (E.). 1962, Schweiz. Milchztg., 88, 443-444.

FlǗckiger (E.). 1964, Schweiz. Milehztg., 90, 1-9.

Flǘriger (E.) et HeUSCher (E.). 1965, Schweiz. Mïlchztg., 91, 3-8.

Foster (E. M.), Nelson (F. E.), Speck (M. L.), Doetsch (R. N.) et Olson (J. C.). 1958, Dairy Microbiology, 165-166 Macmillan et CO LTD London.

Gerhardt (H. T.) et Sommer (M. M.). 1932, J. Dairy Sci., 15, 75.

Girard (R.). 1964, Corr. et Anticor., 12, 347-357.

Herreid (E. O.) et Le Rox Luetscher 1963, J. Dairy Sci., 46, 1309-1319.

Hess (C.). 1951, Rev. Alum. 174, 2 -3.

Holland (R. F.), Shaul (J. D.), Theokas (D, A.) et Windlans (H. M.). 1953, Food Engineer., 25, 75.

JaCkson (C. J.), Howat (G. R.) et Hoar (T. P.). 1936, J. Dairy Res., 7, 284-290. 
Jackson (C. J.), Howat (G. R.) et Hoar (T. P.). 1937, J. Dairy Res., 8, 324-330.

Jensen (J. U.). 1946, J. Dairy Sci., 29, 453-463.

Jensen (J. M.), Trout (G. M.) et Brunner (J. R.). 1963, J. Dairy Sci., 46, 799-809.

JoHns (C. K.). 1954, Canad. J.technol, 32, 71-75.

JоHNS (C. K.). 1962, Dairy Engineer., 79, 156-160.

Johnson (J. J.) et Rolland (C. T.). 1940, J. Dairy Sci., 23, 457-461.

Kaufmann (O. W.), Andrews (R. H.) et Tracy (P. H.). 1955, J, Dairy Sci., 37, 371-379.

Kiermeier (F.), Wildbrett (G.) et Petelkau (G.). 1963, Korrosion, 16, 47-55.

Kiermeier (F.), Wildbrett (G.), Kandler (O.) et Frank (H.). 1965, Die Prüfung von Reinigungs und Desinfektionsmitteln in der Milchwirtschaft, D L G Verlag, Franefort s/Main.

Kiermeter (F.), Grundherr (von K.) et Wildbrett (G.). 1967, Milehw., 22, 76-82.

KoHn-Abrest (E.). 1949, Ann. Fals. Fraudes, 42, 161-165.

KRÜGER (W.), ZÜHLSDORF (M.) et KonRAD (M.). 1962, XVIe Cong. Int. Lait, C, 146-150.

LeClere (E.). 1962, Trib. Cebedeau, 15, 3-10.

Lefaux (R.). 1960, Ind. Alim. Agric., 77, 363-367 et 451-454.

LeWANDOWSKI (T.). 1952, J. Dairy Sci. 35, 449-454.

LEWANDOWSKI (T.). 1954, J. Dairy Sci., 37, 670.

LiNdQvist (B.) et MAGNUSSON (F.). 1963, Svenska Mejerit., 55, 173-174.

Lindevist (B.) et MAGNusson (F.). 1966, XVIIe Cong. Int. Lait., F, 493-495.

Lyster (R. J. L.). 1965, J. Dairy Res., 32, 203-208.

MAJor (W. C. T.). 1962, Queensl. J. Agr. Sci., 19, 107-125.

Merrill (E. P.), Jensen (J. M.) et Bass (S. T.). 1962, J. Dairy Sci., 45, 796- 798.

Meynis de PaUlin (J. J.). 1957, Rev. Alum. 240, 1-2.

Mohr (W.) et Wortmann (A.), 1953, Milchw. Woche Kiel, 8, 111-118.

Монк (W.). 1954, Die Reinigung und Desinfektion in der Milchwirtschaft, Th Mann Hildesheim.

Moore (C. M.). 1944, J. Milk. Technol., 7, 291.

NATHORST (H.). 1950, Jernk Ann., 134, 97-133.

Noe (A.). 1964, Corr, et Anticor., 12, 308-319.

Parker (M. E.). 1941, Milk Pl. Mon., 30, 48.

Peter (N. J.). 1964, Ind. Lait., 206, 16-18.

Poirier (G.). 1963, Corr. et Anticor., 11, 300-304.

Pourbaix (M.), Van Laer (P.), Pourbaix (A.), Van Muydler (J.) et de Zoubov (N.). 1963, Ind. Chim. Belge, 11, 1187-1199.

Resuggan (J. C. L.). 1946, Milk. Ind., 27, 67.

Resuggan (J. C. L.). 1949, J. Soc. Dairy Technol,, 3, 350.

Roderig (H.), Clegg (L. F. .L.), Chapman (H. R.), Rook (J. H. F.) et Hoy (W. A.). 1956, J. Soc. Dairy Technol, 9, 75-80.

RogNer (H.), 1958, Molkerei und Käserei Ztg, 33, 20-25.

Rossman (CHR). 1964, Nickel-Ber., 22, 28-32.

SANderson (L.). 1964, Milk Ind., 54, 32-33.

SANDERSON (L.). 1966, Milk Ind., 58, 26-30.

Schwartze (G. W.), Murphy (F. J.) et Cox (G.J.). 1931, J. Nutrit., 4, 211-213.

Scott (W. I.), Whitmlestone (W. G.) et Lutz (P.). 1962 a, Austr. J. Dairy Technol, 17, 112-115.

Scott (W. I.), Whittlestone (W. G.) et Lutz (P.). 1962 b, Agric. Gaz. N. S. W., 73, 227-229.

Serre (J.) et Laureys (J.). 1963, Corr. et Anticor., 11, 305-312 et 360-367.

SHere (L.). 1942, Food Ind., 14, 44. 
Shewing (J. J.) et Henderson (M. B.). 1952, J. Milk Food Technol., 15, 186.

ShreIr (L. L.). 1963 a, Corrosion, Vol 1, 275, Georges Newnes Ltd., London.

Shreir (L. L.). 1963 b, Corrosion, Vol 1, 251-264, George Newnes Ltd. London.

Summer (W.). 1965, Corr. Technol., 12, 9-11.

Stehle (G.). 1967, Milchw., 22, 374-378.

Swarturng (P.). 1956, XIVe Cong. Int. Lait., 2, 485-491.

Swartuing (P.). 1959, Dairy Sci. Abstr., 21, 1-9.

Szegö (M.) et Keresztessy (A.). 1959, XVe Cong. Int. Lait. 4, 2062-2065.

Szegö (M.) et Keresztessy (A.). 1964, Tejipari Kut. Kölz., 7, 17-23.

Tацвот (J.). 1967, Corr. et Anticor., 15, 43-48.

Thiel (C. C.). 1958, Fmrs' Wkly, 48, no $7: 103-105$; no 8 : 87-88.

Truffert (L.). 1949, Annal. Fals. Fraudes, 42, 151-161.

UHLIG (H. H.). 1963, Corrosion and corrosion control, John Wiley and Sons, New York.

Walters (A. H.) et Vickers (R. H.). 1962, Dairy Engineer, 79, 398-403.

Whittrestone (W. G.). 1958, The principles of mechanical milking, Oswald Ziegler Publ., Sydney.

Whittlestone (W. G.), 1961, Austr. J. Dairy Technol., 16, 230-233.

Whittlestone (W. G.). 1962 a, Austr. J. Dairy Technol., 17, 41-42.

Whittlestone (W. G.). 1962 b, Austr. J. Dairy Technol. 17, 43-44.

Whittlestone (W. G.). 1963, Austr. J. Dairy Technol., 18, 100-103.

Whittlestone (W. G.). 1965, Town Milk, 13, 7-11.

Whittlestone (W. G.). et Lutz (P.). 1962 a, Austr. J. Dairy Technol., 17, 101-103.

Whittlestone (W. G.) et Lutz (P.). 1962 b, Austr. J. Dairy Technol., 17, 202-204.

Whittlestone (W. G.) et Lutz (P.). 1963, Austr. J. Dairy Technol., 18, $173-178$.

Whittlestone (W. G.) et Lutz (P.). 1964, Austr. J. Dairy Technol., 19, 36-40.

Whittlestone (W. G.) et Murrane (D.). 1962, Austr. J. Dairy Technol., 17, 196-201.

Whittlestone (W. G.) et Twomey (A.). 1966, New-Zeal. J. Agri., 161, $51-54$.

Whittlestone (W. G.), Lutz (P.) et Gray (C.). 1964, Austr. J. Dairy Technol., 19, 88-90.

Wild BRETt (G.). 1958, Werlest. und Korr., 9, 521-524.

Wild BRett (G.). 1962, Ztschr. Leben. Unters. und Forsch., 116, 126-134.

Wild BRetT (G.). 1963, Brauw, 16, 397-404.

Wild BRetT (G.). 1964, Deut. Molk., 85, 663-665.

Wildirett (G.). et Kiermeier (F.). 1960, Milchw., 15, 511-519.

Wildobett (G.) et Kiermeier (F.). 1966, XVII e Cong. Int. Lait., EF ; 497-503.

Wildbrett (G.), Kiermeier (F.) et Petelkau (G.). 1961, Werkst. und Korr., 12, 759-764.

Wild brett (G.), Petelkau (G.) et Kiermeier (F.). 1962, Chem. Ing. Technol., 34, 317, 321.

Williams (W. L.) et Eckel (J. F.). 1956, J. Amer. Soc. Naval Eng. 68, 93.

Wolfrum (O. C.). 1962, XVIe Cong. Int. Lait. C, 151-158.

Wotzilk (N.). 1966, XVIIe Cong. Int. Lait. EF, 505-508.

Wrzeclan (M.). 1964, Pratique de l'oxydation anodique, Ed. B.P.I. Paris.

ZÜHLSDORF (M.). 1962 a, Deut. Milchw., 9, 169-170.

ZÜHLSDORF (M.). 1962 b, Deut. Milchw., 9, 263-265. 